\title{
Different Structural Patterns Created by Short Range Variations of Internal Parameters
}

\author{
Flavia Doboga \\ ITT Industries, Washington, U.S.A.
}

\begin{abstract}
This papers presents properties of spatial linear systems described by a certain physical quantity generated by a differential equation. This quantity can be represented by internal electric or magnetic field inside the material, by concentration or by similar physical or chemical quantities. A specific differential equation generates this quantity considering as input the spatial alternating variations of an internal parameter. As a consequence, specific spatial linear variations of the observable output physical quantity appear. It is shown that in case of very short range variations of this internal parameters, systems described by a differential equation able to generate a practical test-function exhibit an output which appears to an external observer under the form of two distinct envelopes. These can be considered as two distinct structural patterns located in the same material along a certain linear axis.
\end{abstract}

Keywords: patterns, short range variations, internal parameters.

\section{Introduction}

This papers presents properties of spatial linear systems described by a certain physical quantity generated by a differential equation. This quantity can be represented by internal electric or magnetic field inside the material, by concentration or by similar physical or chemical quantities. A specific differential equation generates this quantity considering as input the spatial alternating variations of an internal parameter. As a consequence, specific spatial linear variations of the observable output physical quantity appear. It is shown that in case of very short range variations of this internal parameters, systems described by a differential equation able to generate a practical test-function exhibit an output which appears to an external observer under the form of two distinct envelopes. These can be considered as two distinct structural patterns located in the same material along a certain linear axis.

In the ideal mathematical case, suddenly emerging pulses should be simulated using test-functions (functions which differ to zero only on a limited time interval and possessing an infinite number of continuous derivatives on the whole real axis. However, as shown in 1], such test functions, similar to the Dirac functions, can't be generated by a differential equation. The existence of such an equation of evolution, beginning to act at an initial moment of time, would imply the necessity for a derivative of certain order to make a jump at this initial moment 
of time from the zero value to a nonzero value. But this aspect is in contradiction with the property of test-functions to have continuous derivatives of any order on the whole real axis, represented in this case by the time axis. So it results that an ideal test-function can't be generated by a differential equation. For this reason, the analysis must be restricted at practical test-functions 2, defined as functions which differ to zero on a certain interval and possess only a finite number of continuous derivatives on the whole real axis. Mathematical methods based on difference equations are well known [3], but for a higher accuracy of the computer simulation specific Runge-Kutta methods in Matlab are recommended. The physical aspects of dynamical systems able to generate spatial practical test-functions will be studied, for the case when the free-term of the differential equation (corresponding to the internal parameter of material) is represented by alternating functions. The shape of the output signal (obtained by numerical simulations in Matlab based on Runge-Kutta functions) will be analyzed, being shown that for very short range alternating inputs an external observer could notice (in certain condition) the existence of two distinct envelopes corresponding to two distinct structural patterns inside the material. Such aspect differs to the oscillations of unstable type second order systems studied using difference equations [4, and they differs also yo previous studies of the same author [5] where the frequency response of such systems to alternating inputs was studied (in conjunction with the ergodic hypothesis).

\section{Equations Able to Generate Periodical Patterns}

As it is known, a test-function on a spatial interval $[a, b]$ is a function which is nonzero on this interval and which possess an infinite number of continuous derivatives on the whole real axis. For example, the function

$$
\varphi(x)=\left\{\begin{array}{c}
\exp \left(\frac{1}{x^{2}-1}\right) \text { if } x \in(-1,1) \\
0 \text { otherwise }
\end{array}\right.
$$

is a test-function on $[-1,1]$. For a small value of the numerator of the exponent, a rectangular shape of the output is obtained. An example is the case of the function

$$
\varphi(x)=\left\{\begin{array}{cc}
\exp \left(\frac{0.1}{x^{2}-1}\right) \text { if } x \in(-1,1) \\
0 \text { otherwise }
\end{array}\right.
$$

Using the expression of $\varphi(x)$ and of its derivatives of first and second order, a differential equation which admits as solution the function $\varphi$ corresponding to a certain physical quantity can be obtained. However, a test-function can't be the solution of a differential equation. Such an equation of evolution implies a jump at the initial space point for a derivative of certain order, and test-function must possess continuous derivatives of any order on the whole real axis. So it results that a differential equation which admits a test-function $\varphi$ as solution can generate only a practical test-function $f$ similar to $\varphi$, but having a finite number of continuous derivatives on the real $O x$ axis. In order to do this, we must add 
initial conditions for the function $f$ (generated by the differential equation) and for some of its derivatives $f^{(1)}$, and/or $f^{(2)}$ etc. equal to the values of the testfunction $\varphi$ and of some of its derivatives $\varphi^{(1)}$, and/or $\varphi^{(2)}$ etc. at an initial space point $x_{i n}$ very close to the beginning of the working spatial interval. This can be written under the form

$$
f_{x_{i n}}=\varphi_{x_{i n}}, f_{x_{i n}}^{(1)}=\varphi_{x_{i n}}^{(1)} \text { and } / \text { or } f_{x_{i n}}^{(2)}=\varphi_{x_{i n}}^{(2)} \text { etc. }
$$

If we want to generate spatial practical test-functions $f$ which are symmetrical as related to the middle of the working spatial interval, we can choose as space origin for the $O x$ axis the middle of this interval, and so it results that the function $f$ should be invariant under the transformation

$$
x \rightarrow-x
$$

Functions invariant under this transformation can be written in the form $f\left(x^{2}\right)$, (similar to aspects presented in 2]) and so the form of a general second order differential equation generating such functions must be

$$
a_{2}\left(x^{2}\right) \frac{d^{2} f}{d\left(x^{2}\right)^{2}}+a_{1}\left(x^{2}\right) \frac{d f}{d x^{2}}+a_{0}\left(x^{2}\right) f=0
$$

However, for studying the generation of structural pattersns on such a working interval, we must add a free-term, corresponding to the internal parameter of the material (the cause for the variations of the external obersvable physical quntity). Thus, a model for generating a practical test-function using as input the internal parameter $u=u(x), x \in[-1,1]$, is

$$
a_{2}\left(x^{2}\right) \frac{d^{2} f}{d\left(x^{2}\right)^{2}}+a_{1}\left(x^{2}\right) \frac{d f}{d x^{2}}+a_{0}\left(x^{2}\right) f=u
$$

subject to

$$
\lim _{x \rightarrow \pm 1} f^{k}(x)=0 \text { for } k=0,1, \ldots, n .
$$

which are the boundary conditions of a practical test-function. For $u$ represented by aletrnating functions, we should notice periodical variations of the external observable phusical quantity $f$.

\section{Periodical Patterns of Spatial Structures Descibed by Practical Test-Functions}

According to previous considerations for the form of a differential equation invariant at the transformation

$$
x \rightarrow-x
$$

a first order system can be written under the form

$$
\frac{d f}{d\left(x^{2}\right)}=f+u
$$


which converts to

$$
\frac{d f}{d x}=2 x f+2 x u
$$

representing a first order dynamical system. For a periodical input (corresponding to the internal parameter) $u=\sin 10 x$, numerical simulations performed using Runge-Kutta functions in Matlab present an output of an irregular shape (figure 1), not suitable for joining together the outputs for a set of adjoining linear intervals (the value of $f$ at the end of the interval differs in a significant manner to the value of $f$ at the beginning of the interval). A better form for the physical quantity $f$ is obtained for variations of the internal parameter described by the equation $u=\cos 10 x$. In this case the output is symmetrical as related to the middle of the interval (as can be noticed in figure 2) and the results obtained on each interval can be joined together on the whole linear spatial axis, without any discontinuities to appear. The resulting output would be represented by alternances of two great oscillations (one at the end of an interval and another one at the beginning of the next interval) and two small oscillations (around the middle of the next interval).

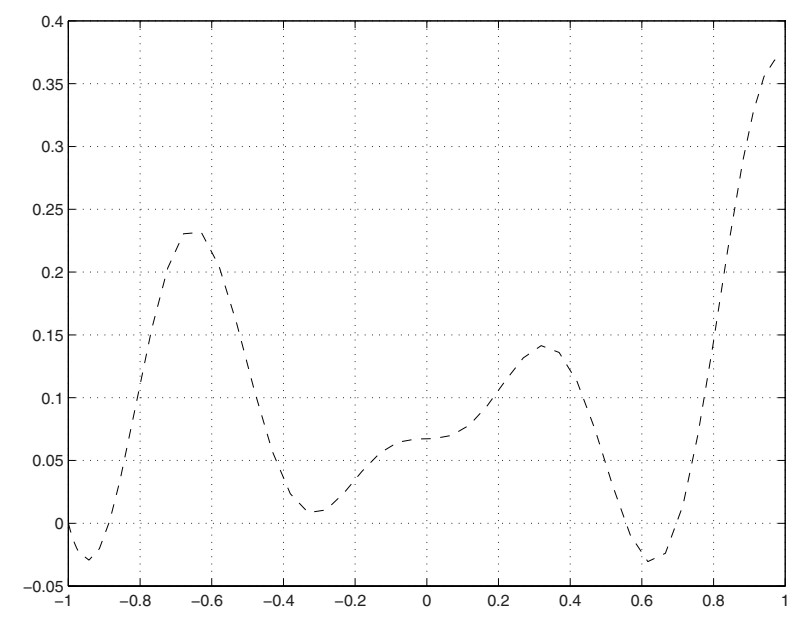

Fig. 1. $f$ versus distance for first order system, input $u=\sin (10 x)$

Similar results are obtained for an undamped dynamical system first order, represented by

$$
\frac{d f}{d\left(x^{2}\right)}=u
$$

which is equivalent to

$$
\frac{d f}{d x}=2 x u
$$




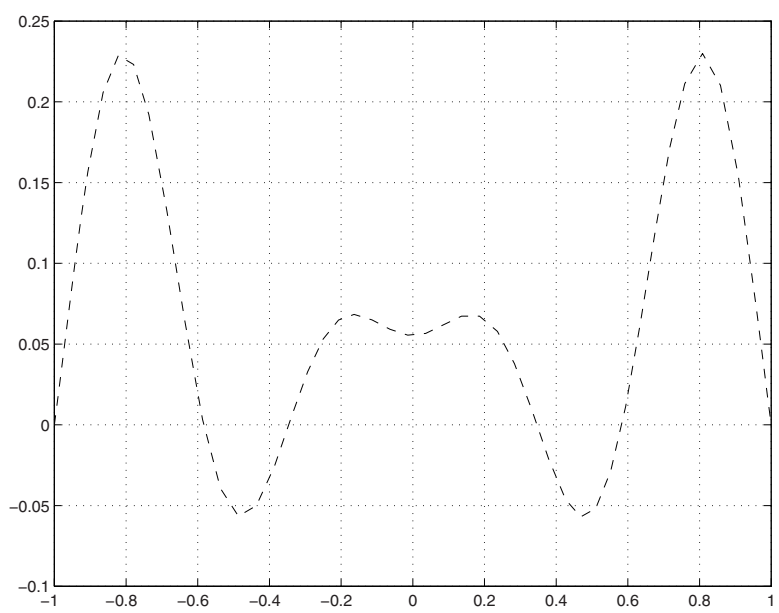

Fig. 2. $f$ versus distance for first order system, input $u=\cos (10 x)$

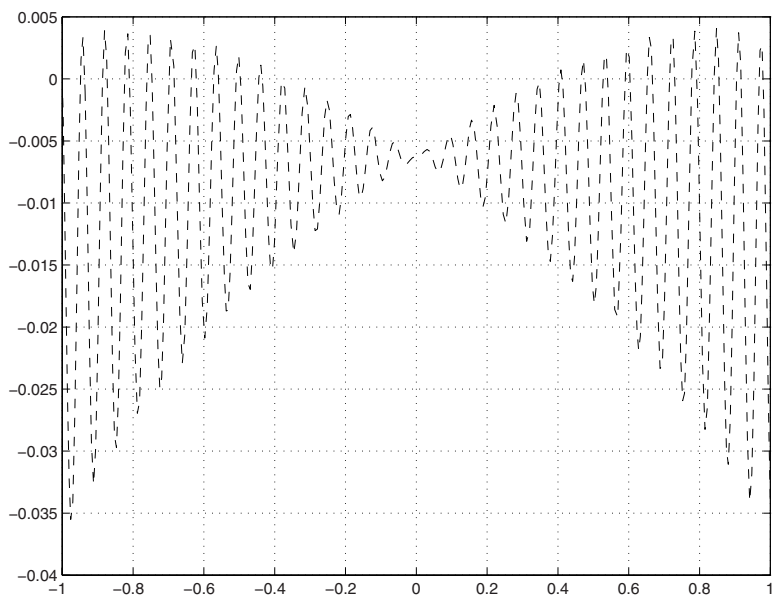

Fig. 3. $f$ versus distance for first order system, input $u=\sin (100 x)$

\section{Connection with the Ergodic Hypothesis}

When the internal parameter presents very short range variations, some new structural patterns can be noticed. Considering an alternating input of the form $u=\sin (100 x)$, it results an observable physical quantity $f$ represented in figure 3; for an alternating cosine input representd by $u=\cos (100 x)$, it results the output $f$ represented in figure 4 . Studying these two graphics, we can notice the presence of two distinct envelopes. Their shape depends on the phase of the 


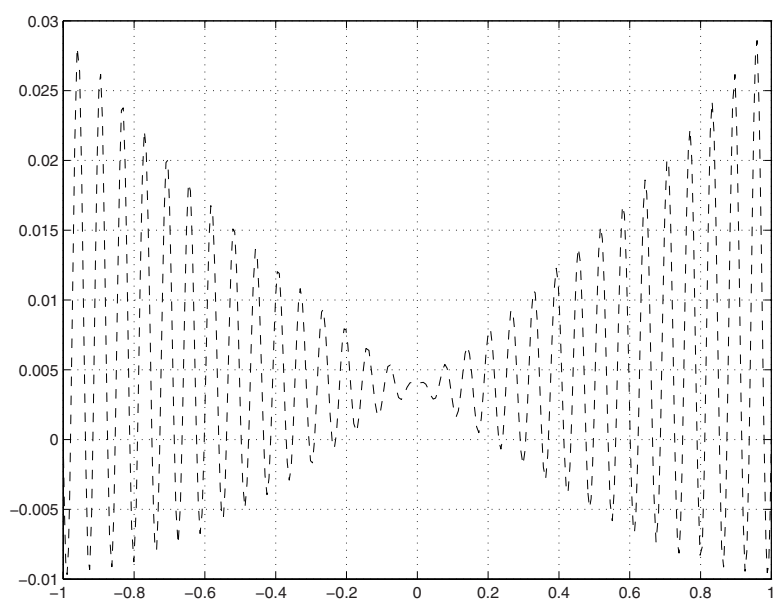

Fig. 4. $f$ versus distance for first order system, input $u=\cos (100 x)$

input alternating component (the internal parameter), as related to the space origin. At first sight, an external observer could notice two distinct functions $f$ inside the same material, along the $O x$ axis. These can be considered as two distinct structural patterns located in the same material, generated by a short range alternating internal parameter $u$ through a certain differential equation (invariant at the transformation $x \rightarrow-x$ ).

\section{Conclusions}

This papers has presented properties of spatial linear systems described by a certain physical quantity generated by a differential equation. A specific differential equation generates this quantity considering as input the spatial alternating variations of an internal parameter. As a consequence, specific spatial linear variations of the observable output physical quantity appear. It was shown that in case of very short range variations of this internal parameters, systems described by a differential equation able to generate a practical test-function exhibit an output which appears to an external observer under the form of two distinct envelopes. These can be considered as two distinct structural patterns located in the same material along a certain linear axis. By this study, a fundamental new interpretation based on spatial aspects has been obtained for graphics previously obtained for non-linear equations of evolution [5] (the novelty of this study being justified).

Acknowledgment. This research work was guided by Cristian Toma (Politehnica University, Bucharest) and Carlo Cattani (University of Salerno, Italy) through a pilot grant of international research involving Politehnica University, 
Salerno University, IBM India Labs and Shanghai University - supported by the National Commission of Romania for UNESCO.

\section{References}

1. Toma, C. : An extension of the notion of observability at filtering and sampling devices, Proceedings of the International Symposium on Signals, Circuits and Systems Iasi SCS 2001, Romania 233-236

2. Toma, G. : Practical test functions generated by computer algorithms, Lecture Notes Computer Science vol. 3482 (2005), 576-584

3. Dzurina, J. : Oscillation of second order differential equations with advanced argument, Math. Slovaca, 453 (1995) 263-268

4. Zhang, Zh., Ping, B., Dong, W. : Oscillation of unstable type second order nonlinear difference equation, Korean J. Computer and Appl. Math. 91 (2002) 87-99

5. Doboga, F., Toma, G., Pusca, St., Ghelmez, M., Morarescu, C. : Filtering Properties of Practical Test Functions and the Ergodic Hypothesis, Lecture Notes Computer Science vol. 3482 (2005), 563-568 